\title{
ERGODIC PROJECTIONS OF CONTINUOUS AND DISCRETE SEMIGROUPS
}

\author{
SEN-YEN SHAW
}

\begin{abstract}
Let $X$ be a Banach space. Let $\{T(t) ; t>0\}$ be a uniformly bounded semigroup of operators on $X$, which converges strongly to $P$, known to be a projection, as $t$ goes to 0 . If $A$ is its generator and $X_{0}$ [resp., $X_{t}, t>0$ ] is the set of $x$ for which

$$
P_{0} x \equiv \lim _{t \rightarrow \infty} t^{-1} \int_{0}^{t} T(\tau) x d \tau \quad\left[\text { resp., } P_{t} x \equiv \lim _{n \rightarrow \infty} n^{-1} \sum_{i=0}^{n-1} T(i t) x\right]
$$

exists, then, for each $t>0, P_{t}$ is a bounded projection in $X_{t}$; when $t=0$, $X_{0}=N(A) \oplus \overline{R(A)} \oplus N(P), R\left(P_{0}\right)=N(A)$ and $N\left(P_{0}\right)=\overline{R(A)} \oplus N(P)$; when $t>0$, then

$$
X_{t}=N(T(t)-I) \oplus \overline{R(T(t)-I)},
$$

$R\left(P_{t}\right)=N(T(t)-I)$ and $N\left(P_{t}\right)=\overline{R(T(t)-I)} ; X_{t}=X$ for all $t>0$ if $X$ is reflexive. Some results on relations among the projections $P_{t}, t>0$, are obtained. In particular, we have $P_{t}=P_{0}$ for all sufficiently small $t$ if $A$ is bounded.
\end{abstract}

1. Introduction. Let $S=\{T(t) ; t>0\}$ be a semigroup of bounded linear operators on a Banach space $X$. The infinitesimal generator $A$ of $S$ is defined as $A x \equiv \lim _{t \rightarrow 0}[T(t)-I] x / t$ wherever the limit exists. Let $N(A)$ and $R(A)$ denote the null space and range, respectively, of $A$. Also we denote by $R(S)$ the set $\{T(t) x ; x \in X, t>0\}$.

If $S$ satisfies the additional assumption that

$$
P x=\lim _{t \rightarrow 0} T(t) x \quad \text { exists for all } x \in X,
$$

then $P$ is a projection from $X$ onto $\overline{R(S)}$, and $T(t)=P T(t)=T(t) P$ (see [3, p. 319]). Thus, we have $T(t)|\overline{R(S)}=T(t) P, T(t)| N(P)=0$ and

$$
T(t)=\left[\begin{array}{ll}
T(t) \mid \overline{R(S)} & 0 \\
0 & 0
\end{array}\right]
$$

$T(t)$ is strongly continuous; in fact, $T(t) \mid \overline{R(S)}$ is a semigroup of class $\left(C_{0}\right)$ in $\overline{R(S)}$ with generator $A \mid \overline{R(S)}=A$. It follows that $A$ is a closed operator with $\overline{D(A)}=\overline{R(S)}$ and $R(A) \subset \overline{R(S)}$. The following extension of Theorem 1 with Corollary 2 of [4] (or cf. [2]) is obtained immediately.

Received by the editors August 4, 1978 and, in revised form, January 5, 1979.

AMS (MOS) subject classifications (1970). Primary 47D05, 47A35.

Key words and phrases. Semigroups of operators, mean ergodic theorem, ergodic projections, infinitesimal generator. 
THeOREM 1.1. If $S$ is bounded by $M \geqslant 1$ and if it satisfies (1), then the set $X_{0}$ of all $x \in X$ such that

$$
P_{0} x \equiv \lim _{t \rightarrow \infty} t^{-1} \int_{0}^{t} T(\tau) x d \tau=\lim _{\lambda \rightarrow 0^{+}} \lambda(\lambda I-A)^{-1} P x
$$

exists is a closed subspace; $X_{0}=N(A) \oplus \overline{R(A)} \oplus N(P)$. $P_{0}$ is a projection in $X_{0}$, with range $N(A)$ and null space $\overline{R(A)} \oplus N(P)$, and has norm $\left\|P_{0}\right\| \leqslant M$. In general, $N(A) \oplus \overline{R(A)} \subset \overline{R(S)}$; but, if $X$ is reflexive, then they are identical and therefore $X_{0}=X$.

Using the notation $A_{t} \equiv[T(t)-I] / t$, we have $\left\|\exp \left(\tau A_{t}\right)\right\| \leqslant M$ for all $t>0$ and for all $\tau \geqslant 0$ if $\|T(t)\| \leqslant M$ for all $t>0$. (See Corollary 2.2.) Now, by Theorem 1.1, we can associate with each $t>0$ a closed subspace $X_{t} \equiv$ $N\left(A_{t}\right) \oplus \overline{R\left(A_{t}\right)}$ and a projection $P_{t}: X_{t} \rightarrow X_{t}$ which is defined as

$$
P_{t} x \equiv \lim _{u \rightarrow \infty} \frac{1}{u} \int_{0}^{u} \exp \left(\tau A_{t}\right) x d \tau, \quad x \in X_{t} .
$$

We have $R\left(P_{t}\right)=N\left(A_{t}\right), N\left(P_{t}\right)=\overline{R\left(A_{t}\right)}$ and $\left\|P_{t}\right\| \leqslant M$.

An interesting phenomenon is that for each $t>0, P_{t}$, defined as above, is precisely the same as that defined by

$$
P_{t} x=\lim _{n \rightarrow \infty} \frac{1}{n} \sum_{i=0}^{n-1} T(i t) x .
$$

This results from Theorem 1 of $[6$, p. 213] on the limit problem

$$
J x \equiv \lim _{n \rightarrow \infty} \frac{1}{n} \sum_{i=0}^{n-1} T^{i} x
$$

for a general uniformly bounded discrete semigroup $\left\{T^{n} ; n=0,1, \ldots\right\}$. Actually, this can also be derived from Theorem 1.1, which will be done in \$2. \$3 is concerned with relations among the projections $P_{t}, t \geqslant 0$.

2. Ergodic theorems for discrete semigroups. Let $T \in B(X)$. Its spectral radius $r_{\sigma}(T) \equiv \max \{|\lambda| ; \lambda \in \sigma(T)\}$ is equal to $\lim _{n \rightarrow \infty}\left(\left\|T^{n}\right\|\right)^{1 / n}<\|T\|$. Hence, for any $r>r_{\sigma}(T)$, there is an $M \geqslant 1$ such that $\left\|T^{n}\right\| \leqslant M r^{n}$ for all $n=0,1,2, \ldots$.

Conversely, we have

LEMMA 2.1. If $\left\|T^{n}\right\| \leqslant M r_{0}^{n}, n=0,1,2, \ldots$, then every $\lambda$ with $|\lambda|>r_{0}$ belongs to the resolvent set $\rho(T)$, i.e., $r_{\sigma}(T)<r_{0}$, and the following inequality holds.

$$
\|\exp (t T)\| \leqslant M \exp \left(t r_{0}\right), \quad t>0
$$

The proof is just routine arguments on the convergence of the Neumann series and the norm estimate of the series expansion of $\exp (t T)$. The details will be omitted here.

If we replace $t, T$ and $r_{0}$ by $t / \tau, T(\tau)$ and $e^{w \tau}$ respectively, then we have 
COROLlaRY 2.2. If $\|T(t)\| \leqslant M e^{w t}$ for all $t>0$, then

$$
\left\|\exp \left(t A_{\tau}\right)\right\| \leqslant M \exp \left(t \frac{e^{w \tau}-1}{\tau}\right)
$$

for all $\tau>0$ and for all $t \geqslant 0$.

Our key result in this section is

THEOREM 2.3. If $\left\|T^{n}\right\| \leqslant M$ for some $M \geqslant 1$ and all $n=0,1,2, \ldots$, then the limit $\lim _{n \rightarrow \infty}(1 / n) \sum_{i=0}^{n-1} T^{i} x$ exists if and only if the limit $\lim _{\lambda \rightarrow 0^{+}} \lambda[\lambda I-$ $(T-I)]^{-1} x$ exists, and they are identical when they exist.

The proof uses the following:

Proposition. Let $f(t)$ be a bounded and strongly measurable $X$-valued function defined on $(0, \infty)$. Then

$$
\lim _{t \rightarrow \infty} \frac{1}{t} \int_{0}^{t} f(\tau) d \tau=\lim _{\mu \rightarrow 0^{+}} \mu \int_{0}^{\infty} e^{-\mu t} f(t) d t
$$

provided either limit exists.

For the proof, see [5, Theorems 8.2.3 and 8.2.4].

Proof of Theorem 2.3. We put $f(t)=T^{i} x$ for $i<t \leqslant i+1, i=0,1, \ldots$ Then the following computation verifies the assertion.

$$
\begin{aligned}
\lim _{n \rightarrow \infty} \frac{1}{n} \sum_{i=0}^{n-1} T^{i} x & =\lim _{n \rightarrow \infty} \frac{1}{n} \int_{0}^{n} f(t) d t=\lim _{\mu \rightarrow 0^{+}} \mu \int_{0}^{\infty} e^{-\mu t} f(t) d t \\
& =\lim _{\mu \rightarrow 0^{+}} \mu \sum_{i=0}^{\infty} \int_{i}^{i+1} e^{-\mu} T^{i} x d t \\
& =\lim _{\mu \rightarrow 0^{+}} \sum_{i=0}^{\infty}\left[e^{-i \mu}-e^{-(i+1) \mu}\right] T^{i} x \\
& =\lim _{\mu \rightarrow 0^{+}}\left(e^{\mu}-1\right) \sum_{i=0}^{\infty} e^{-(i+1) \mu} T^{i} x \\
& =\lim _{\mu \rightarrow 0^{+}}\left(e^{\mu}-1\right)\left(e^{\mu} I-T\right)^{-1} x \\
& =\lim _{\lambda \rightarrow 0^{+}} \lambda[\lambda I-(T-I)]^{-1} x .
\end{aligned}
$$

The invertibility of $\lambda I-(T-I)$ follows from Lemma 2.1 .

Since $\left\|T^{n}\right\| \leqslant M$ implies $\|\exp (t(T-I))\| \leqslant M$ for all $t \geqslant 0$, Theorem 1.1 applies with $A=T-I$ and $P=I$ so that the limits in Theorem 2.3 are also equal to the $\operatorname{limit}_{\lim _{t \rightarrow \infty}}(1 / t) \int_{0}^{t} e^{\tau(T-I)} x d \tau$. Therefore we have the following mean ergodic theorem for discrete semigroups. 
THEOREM 2.4. If $\left\|T^{n}\right\| \leqslant M$ for all $n=0,1,2, \ldots$, then the set $X_{J}$ of all $x \in X$ such that

$$
\begin{aligned}
J x & \equiv \lim _{n \rightarrow \infty} \frac{1}{n} \sum_{i=0}^{n-1} T^{i} x=\lim _{\lambda \rightarrow 0^{+}} \lambda[\lambda I-(T-I)]^{-1} x \\
& =\lim _{t \rightarrow \infty} \frac{1}{t} \int_{0}^{t} e^{\tau(T-I)} x d \tau
\end{aligned}
$$

exists is $N(T-I) \oplus \overline{R(T-I)}$. $J$ is a projection in $X_{J}$ with range $N(T-I)$, null space $\overline{R(T-I)}$, and with norm $\|J\| \leqslant M$. When $X$ is reflexive, we have $X_{J}=X$.

3. The projections $P_{t}$. Throughout this section, let $S=\{T(t) ; t>0\}$ satisfy the assumptions in Theorem 1.1, and let $\left\{P_{t} ; t \geqslant 0\right\}$ be the projections defined by (2) and (3). That is, if $t=0$, then $X_{0} \equiv N(A) \oplus \overline{R(A)} \oplus N(P)$, $R\left(P_{0}\right)=N(A)$ and $N\left(P_{0}\right)=\overline{R(A)} \oplus N(P)$; if $t>0$, then $X_{t} \equiv N\left(A_{t}\right) \oplus \overline{R\left(A_{t}\right)}, R\left(P_{t}\right)=N\left(A_{t}\right)$ and $N\left(P_{t}\right)=\overline{R\left(A_{t}\right)}$. In this section we will study relations among $P_{t}$.

We begin with three lemmas which will be used in the proofs of the subsequent theorems.

LEMMA 3.1. $x$ belongs to $X_{0}$ if and only if $\lim _{n \rightarrow \infty}\left(1 / t_{n}\right) \int_{0}^{t_{n}} T(\tau) x d \tau$ exists for some increasing sequence of positive numbers $\left\{t_{n}\right\}$ which diverges to infinity and satisfies the condition that $a=\sup _{n}\left(t_{n+1}-t_{n}\right)<\infty$. For any such $x$ and $\left\{t_{n}\right\}$, the limit is $P_{0} x$.

In particular, the assertion holds when $t_{n}=n t$, which is the case we will need in the proof of Theorem 3.4.

Proof. The necessity is trivial. To show the sufficiency, let $\left\{t_{n}\right\}$ satisfy the required condition and $y$ be the limit. Since for any $t$, there is an integer $n$ such that $t_{n-1} \leqslant t \leqslant t_{n}$, therefore, we have the estimate

$$
\begin{aligned}
\| \frac{1}{t} & \int_{0}^{t} T(s) x d s-\frac{1}{t_{n}} \int_{0}^{t_{n}} T(s) x d s \| \\
& <\left|\frac{t_{n}}{t}-1\right|\left\|\frac{1}{t_{n}} \int_{0}^{t_{n}} T(s) x d s\right\|+\left\|\frac{1}{t} \int_{t}^{t_{n}} T(s) x d s\right\| \\
& <2 \frac{\left(t_{n}-t\right)}{t} M\|x\| \leqslant \frac{2 a M\|x\|}{t}
\end{aligned}
$$

which goes to 0 as $t \rightarrow \infty$. This means that $x \in X_{0}$ and $y=P_{0} x$.

LEMMA 3.2. The following statements are equivalent.

(a) $x \in N(A)$.

(b) $T(t) x=x$ for all $t>0$.

(c) $T(t) x=x$ for all $t$ in some infinite set $U$ of positive numbers which has at least one limit point.

(d) There is a positive sequence $\left\{t_{n}\right\}$ such that $t_{n} \rightarrow 0$ and such that $T\left(t_{n}\right) x=$ $x$ for all $n$. 
Proof. (a) $\Rightarrow$ (b). If $x \in N(A) \subset \overline{R(S)}=R(P)$, then $d T(t) x / d t=T(t) A x$ $=0$ for all $t>0$. So,

$$
\begin{aligned}
T(t) x-x & =T(t) x-P x=\lim _{\varepsilon \rightarrow 0^{+}}[T(t) x-T(\varepsilon) x] \\
& =\lim _{\varepsilon \rightarrow 0^{+}} \int_{\varepsilon}^{t} \frac{d}{d \tau} T(\tau) x d \tau=0
\end{aligned}
$$

for all $t>0$. "(b) $\Rightarrow$ (a)" follows from the definition of $A$, and "(b) $\Rightarrow$ (c)" is trivial. To prove (c) $\Rightarrow$ (d), let $\left\{s_{n}\right\} \subset U$ and $s_{n} \rightarrow s_{0}>0$. Then, $T\left(s_{0}\right) x=$ $\lim _{n \rightarrow \infty} T\left(s_{n}\right) x=x$ by the strong continuity of $T(t)$. On taking $t_{n}=\left|s_{n}-s_{0}\right|$, we have $t_{n} \rightarrow 0$ and $T\left(t_{n}\right) x=x, n=1,2, \ldots$ In fact, if $s_{n}>s_{0}$, then

$$
T\left(t_{n}\right) x=T\left(t_{n}\right) T\left(s_{0}\right) x=T\left(s_{n}\right) x=x ;
$$

if $s_{n}<s_{0}$, then $T\left(t_{n}\right) x=T\left(t_{n}\right) T\left(s_{n}\right) x=T\left(t_{n}+s_{n}\right) x=T\left(s_{0}\right) x=x$. Next, suppose (d) holds. Since the set of positive $t$ such that $T(t) x=x$ is a relatively closed sub-semigroup of $(0, \infty)$, and since it contains arbitrarily small numbers, it is all of $(0, \infty)$, i.e., (b) is true.

LEMMA 3.3. If $C_{t}$ denotes the operator $t^{-1} \int_{0}^{t} T(\tau) d \tau$, then

$$
C_{n t} x=C_{t} \frac{1}{n} \sum_{i=0}^{n-1} T(i t) x=\left[\frac{1}{n} \sum_{i=0}^{n-1} T(i t)\right] C_{t} x
$$

for all $x \in X, t>0$ and $n=1,2, \ldots$

Proof.

$$
\begin{aligned}
C_{n t} x & =\frac{1}{n} \int_{0}^{n} T(s t) x d s=\frac{1}{n} \sum_{i=0}^{n-1} \int_{i}^{i+1} T(s t) x d s \\
& =\frac{1}{n} \sum_{i=0}^{n-1} T(i t) \int_{0}^{1} T(s t) x d s \\
& =\left[\frac{1}{n} \sum_{i=0}^{n-1} T(i t)\right] C_{t} x,
\end{aligned}
$$

and similarly for the other identity.

The following theorems and corollaries form the main results of this section.

THEOREM 3.4. The following relations hold for all $t>0$.

(i) $X_{t} \subset X_{0}, N(P) \subset N\left(P_{t}\right) \subset N\left(P_{0}\right)$ and $R\left(P_{t}\right) \supset R\left(P_{0}\right)$.

(ii) $C_{t} X_{0} \subset X_{t}, C_{t} N\left(P_{0}\right) \subset N\left(P_{t}\right), C_{t}\left|R\left(P_{t}\right)=P_{0}\right| R\left(P_{t}\right)$ and $C_{t} \mid R\left(P_{0}\right)=I$.

(iii) $P_{0} X_{t} \subset X_{t}, P_{0}=P_{t} C_{t} \mid X_{0}$ and $P_{0}\left|X_{t}=P_{t} C_{t}\right| X_{t}=C_{t} P_{t}$.

Proof. All the assertions follow from the two identities:

$$
\begin{aligned}
& P_{0} x=P_{t} C_{t} x \text { for all } x \in X_{0}, \\
& P_{0} x=P_{t} C_{t} x=C_{t} P_{t} x \text { for all } x \in X_{t} .
\end{aligned}
$$

But these are obtained by taking limits of terms in (6). 
Corollary 3.5. $R\left(P_{t}\right)=R\left(P_{0}\right) \oplus\left[R\left(P_{t}\right) \cap N\left(P_{0}\right)\right]$. If $N\left(P_{t}\right)=N\left(P_{0}\right)$, then $R\left(P_{t}\right)=R\left(P_{0}\right)$, and so, $X_{t}=X_{0}$ and $P_{t}=P_{0}$.

From the fact that $T(t) x=x$ for all $x \in N(A)$, and $T(t) A x=A T(t) x$ for all $x \in D(A)$, we see that $N(A), \overline{R(A)}$ and $X_{0}=N(A) \oplus \overline{R(A)}$ are invariant under $T(t)$ and so under $C_{t}$, and that $C_{t} \mid \overline{R(A)}$ is a surjection if and only if $C_{t} \mid X_{0}$ is. Now, if they are surjective, then

$$
\overline{R(A)}=C_{t} \overline{R(A)}=C_{t}[\overline{R(A)} \oplus N(P)]=C_{t} N\left(P_{0}\right) \subset N\left(P_{t}\right) .
$$

This, with $N(P) \subset N\left(P_{t}\right)$, yields the relation $N\left(P_{0}\right)=\overline{R(A)} \oplus N(P) \subset N\left(P_{t}\right)$ $\subset N\left(P_{0}\right)$. Therefore, from Corollary 3.5 follows the next

Corollary 3.6 If $C_{t} \mid \overline{R(A)}$ [or $\left.C_{t} \mid X_{0}\right]$ is surjective, then $X_{t}=X_{0}$ and $P_{t}=P_{0}$.

COROllary 3.7. If $\|T(t)-P\| \rightarrow 0$ as $t \rightarrow 0$ (which is equivalent to saying that the generator $A$ is bounded), then there is a $\delta>0$ such that $X_{t}=X_{0}$ and $P_{t}=P_{0}$ for all $0 \leqslant t<\delta$.

Proof. We have $\|T(t) \mid \overline{R(A)}-I\| \rightarrow 0$ as $t \rightarrow 0$. Let $\delta>0$ be so chosen that $0<t<\delta$ implies $\|T(t) \mid \overline{R(A)}-I\|<1$. Then for all such $t$,

$$
\left\|C_{t} \mid \overline{R(A)}-I\right\|=\left\|t^{-1} \int_{0}^{t}[T(\tau) \mid \overline{R(A)}-I] d \tau\right\|<1
$$

Thus, $C_{t} \mid \overline{R(A)}$ is invertible, and therefore surjective. The conclusion follows immediately from Corollary 3.6.

Remarks AND EXAMPLes. (a) If $X$ is reflexive, then $N\left(P_{t}\right)=N\left(P_{0}\right)$ if and only if $R\left(P_{t}\right)=R\left(P_{0}\right)$.

(b) Here is an example for Corollary 3.7. Let $T(t)$ be the multiplication by $e^{i t x}$ on $C[0,1]$. Computations show

$$
\begin{aligned}
&\left(C_{t} f\right)(x) \equiv \frac{1}{t} \int_{0}^{t} e^{i \tau x} f(x) \\
&= \begin{cases}f(0) & \text { if } x=0 \\
f(x)\left(e^{i t x}-1\right) / i t x & \text { if } x \neq 0\end{cases} \\
& f_{n}(x) \equiv \frac{1}{n} \sum_{k=0}^{n-1} e^{i k t x} f(x) \\
&=\left\{\begin{array}{lll}
f(x) & \text { if } t x \equiv 0 \quad(\bmod 2 \pi), \\
f(x) \exp \left(i \frac{1}{2}(n-1) t x\right)\left(\sin \frac{1}{2} n t x\right) / n \sin \frac{1}{2}(t x) & \text { elsewhere. }
\end{array}\right.
\end{aligned}
$$

(7) tells us that if $C_{t} f$ converges in $\|\cdot\|_{\infty}$ while $t \rightarrow \infty$, then $f(0)$ has to be 0 and the limit is the zero function. Hence we have $R\left(P_{0}\right)=\{0\}$ and $X_{0}=$ $N\left(P_{0}\right) \subset\{f \in C[0,1] ; f(0)=0\}$. This inclusion actually is an equality. In fact, let $f$ be continuous and $f(0)=0$. Then, for given $\varepsilon>0$, there is a $\delta>0$ such that $|f(x)|<\varepsilon$ on $[0, \delta)$, which implies $\left|\left(C_{t} f\right)(x)\right|<\varepsilon$ for $x \in[0, \delta)$. If 
$t>2\|f\|_{\infty} / \delta \varepsilon$, then

$$
\left|\left(C_{t} f\right)(x)\right| \leqslant\left|\left(e^{i t x}-1\right) / i t x\right||f(x)| \leqslant 2\|f\|_{\infty} / t \delta<\varepsilon
$$

for $x$ in $[\delta, 1]$ also. Thus $\left\|C_{t} f\right\| \rightarrow 0$ as $\rightarrow \infty$, i.e., $f \in N\left(P_{0}\right)$.

Similarly, one can show without difficulty that $\left\{f_{n}\right\}$ converges uniformly if and only if $f(x)=0$ for all those $x$ such that $t x \equiv 0(\bmod 2 \pi)$, and that the limit is 0 if it exists. That is, $R\left(P_{t}\right)=\{0\}$ and $X_{t}=N\left(P_{t}\right)=\{f \in C[0,1]$; $f(x)=0$ if $t x \equiv 0(\bmod 2 \pi)\}$. We have $X_{t}=X_{0}$ and $P_{t}=P_{0}$ for $0 \leqslant t<2 \pi$, but $X_{2 \pi}=N\left(P_{2 \pi}\right)=\{f \in C[0,1] ; f(0)=f(1)=0\} \varsubsetneqq X_{0}$.

(c) The condition $\|T(t)-P\| \rightarrow 0$ is not a necessity in Corollary 3.7. For instance, the semigroup of left translations $T(t): f(x) \rightarrow f(x+t)$ on $X=$ $L^{p}(-\infty, \infty), 1<p<\infty$, is not continuous in operator norm, but we have $X_{t}=X_{0}=X$ and $P_{t}=P_{0}=0$ for all $t>0$. First, $R\left(P_{0}\right)=N(A)=\{0\}$ since the spectrum of $A=d / d x$ is purely continuous [1, p. 37]; secondly, $R\left(P_{t}\right)=$ $N(T(t)-I)=\{0\}$ since the only periodic (a.e.) function in $L^{p}(1<p<\infty)$ is 0 ; finally, remark (a) applies.

(d) There are semigroups (with unbounded generators) such that $P_{t} \neq P_{0}$ for every $t>0$. For instance, for the semigroup of multiplications by $e^{i t x}$ on $\mathrm{UCB}(-\infty, \infty)$, the set of bounded uniformly continuous functions on $(-\infty, \infty)$, we have $R\left(P_{t}\right)=R\left(P_{0}\right)=\{0\}, X_{0}=N\left(P_{0}\right)=\{f \in \mathrm{UCB}(-\infty, \infty)$; $f(0)=0\}$ and $X_{t}=N\left(P_{t}\right)=\{f \in \operatorname{UCB}(-\infty, \infty) ; f(2 k \pi / t)=0, k=$ $0, \pm 1, \pm 2, \ldots\}$ for $t>0$. Another example is the left translations on $\mathrm{UCB}(-\infty, \infty)$, for which we have $R\left(P_{0}\right)=N(A)=$ \{constant functions $\}$ and $R\left(P_{t}\right)=N(T(t)-I)=\{$ continuous periodic functions with period $t\}$.

For general strongly continuous semigroups, we have

THEOREM 3.8. If $\left\{t_{n}\right\} \rightarrow 0$, then $\cap_{n=1}^{\infty} R\left(P_{t_{n}}\right)=R\left(P_{0}\right)$ and $\overline{\operatorname{span}}\left\{N\left(P_{t_{n}}\right)\right.$; $n=1,2, \ldots\}=N\left(P_{0}\right)$.

This follows from the next theorem plus the fact that $N\left(P_{t}\right) \subset N\left(P_{0}\right)$ for all $t>0$.

THEOREM 3.9. Let $U$ be an infinite set of nonnegative numbers with at least one limit point. Then,

(i) $\cap\left\{R\left(P_{t}\right) ; t \in U\right\}=R\left(P_{0}\right)$,

(ii) $\left[\cup\left\{N\left(P_{t}\right) ; t \in U\right\}\right]^{-}=\left[\cup\left\{N\left(P_{t}\right) ; t \in \bar{U}\right\}\right]^{-}$.

Proof. $x \in R\left(P_{t}\right)=N(T(t)-I)$ for all $t \in U$ implies $x \in N(A)=$ $R\left(P_{0}\right)$, by Lemma 3.2, therefore $\cap\left\{R\left(P_{t}\right) ; t \in U\right\} \subset R\left(P_{0}\right)$. But we also have $R\left(P_{0}\right) \subset R\left(P_{t}\right)$ for all $t$. So, (i) is true.

To show (ii), it suffices to show that the left side of it contains $N\left(P_{t_{0}}\right)$ for any limit point $t_{0}$ of $U$. Suppose $\left\{t_{n}\right\} \subset U$ and $t_{n} \rightarrow t_{0}$. If $t_{0}>0$, then, for any $x \in N\left(P_{t_{0}}\right)=R\left(T\left(t_{0}\right)-I\right)^{-}$, there are $\left\{y_{n}\right\} \subset X$ such that

$$
\begin{aligned}
x & =\lim _{m \rightarrow \infty}\left(T\left(t_{0}\right)-I\right) y_{m} \\
& =\lim _{m \rightarrow \infty} \lim _{n \rightarrow \infty}\left(T\left(t_{n}\right)-I\right) y_{m} \in\left[\bigcup\left\{N\left(P_{t_{n}}\right) ; n=1,2, \ldots\right\}\right]^{-} .
\end{aligned}
$$


If $t_{0}=0$, then for any $x \in N\left(P_{0}\right)=\overline{R(A)} \oplus N(P)$, there are $u \in N(P)$ and $\left\{w_{n}\right\} \subset D(A)$ such that

$$
\begin{aligned}
x & =u+\lim _{m \rightarrow \infty} A w_{m}=u+\lim _{m \rightarrow \infty} \lim _{n \rightarrow \infty}\left(T\left(t_{n}\right)-I\right) w_{m} / t_{n} \\
& =\lim _{m \rightarrow \infty} \lim _{n \rightarrow \infty}\left(T\left(t_{n}\right)-I\right)\left(w_{m} / t_{n}-u\right) \in\left[\bigcap\left\{N\left(P_{t_{n}}\right) ; n=1,2, \ldots\right\}\right]^{-} .
\end{aligned}
$$

Hence, we always have $N\left(P_{t_{0}}\right) \subset\left[\cup\left\{N\left(P_{t}\right) ; t \in U\right\}\right]^{-}$once $t_{0}$ belongs to $\bar{U}$. This ends the proof.

\section{REFERENCES}

1. P. L. Butzer and H. Berens, Semi-groups of operators and approximation, Springer-Verlag, New York, 1967.

2. J. A. Goldstein, C. Radin and R. E. Showalter, Convergence rates of ergodic limits for semigroups and cosine functions, Semigroup Forum 16 (1978), 89-95.

3. E. Hille and R. S. Phillips, Functional analysis and semigroups, Amer. Math. Soc. Colloq. Publ., vol. 31, Amer. Math. Soc., Providence, R. I., 1957.

4. S. C. Lin and S. Y. Shaw, Ergodic theorems of semigroups and application, Bull. Inst. Math. Academia Sinica 6 (1978). 181-188.

5. D. V. Widder, An introduction to transform theory, Academic Press, New York, 1971.

6. K. Yosida, Functional analysis, Springer-Verlag, New York, 1970.

Department of Mathematics, National Central University, Chung-Li, Tatwan 320, REPUBLIC OF ChINA 